\title{
Energy Efficient Scheduling for Mobile Push Notifications
}

\author{
Utku Günay Acer, Afra Mashhadi, Claudio Forlivesi, Fahim Kawsar \\ Bell Laboratories, Alcatel-Lucent \\ \{utkuacer, afra.mashhadi, claudio.forlivesi, fahim.kawsar\}@bell-labs.com
}

\begin{abstract}
Push notifications are small and succinct messages used by mobile applications to inform users of new events and updates. These notifications are pushed to the user devices by a set of dedicated notification servers (e.g., Apple Push Notification Server, Google Cloud Messaging Server, etc.) as they arrive from the content providers of the mobile applications. However, due to their intrinsic small size and sporadic nature, the transfer of these messages is not power efficient, especially on cellular networks. To address this, we propose a network centric scheduling mechanism that delays the delivery of these messages as appropriate by sensing and predicting users' cellular network activities. A trace based evaluation with 60 users' cellular network logs of 30 days shows that we can reduce the energy consumption of mobile devices by $10 \%$ for an average delay of 150 seconds in notification delivery. As a network based system that does not require any modifications to user devices, scheduling push notifications opens up interesting opportunities for mobile operators to provide value added and differentiating services, especially considering the sharp rise of non-critical push notification messages.
\end{abstract}

\section{Categories and Subject Descriptors}

H.4 [Information Systems Applications]: Communications Applications

\section{Keywords}

Push Notification, Network Sensing, Activity Prediction, Energy Efficiency

\section{INTRODUCTION}

The push notifications have become increasingly prevalent and part of many smartphones applications, allowing the third party content providers to initiate communication with the users even when the application is not actively being used. This type of communication provides a visual or audible cue to inform the mobile users about new unattended short messages or event updates. These messages have become so popular that users now receive hundreds of push notifications per day, many of which are non critical and does not require immediate attention (excluding SMS, and messages from other real time communication services, e.g., Skype, WhatsApp, etc.) $[13,15]$. While the impact of this increase has been studied in terms of its disruptive aspects on the end users, little attention has been given to the push notification delivery services from a system perspective and their impact on energy consumption on the mobile device.

The push notification messages are often small in size and sporadic in nature. Once issued by the third party content provider, these messages are sent to the push notification servers, such as Apple Push Notification service (APNs) or Google Cloud Messaging (GCM) which then transfers this information to the user device. The current implementation of push notification services is based on store-and-forward mechanism, pushing any notifications to the device as soon as the device is reachable. However, these services do not account for the devices' network interfaces and corresponding impact on the energy consumption. For instance, the cellular network interfaces in mobile phones do not immediately go into the low power idle-state after a network operation, instead, they remain in the high-power state for a certain duration, often referred to as tail time. Hence, the delivery of small messages instantly and in isolation, as currently is done for push notification messages, is an inefficient method which consumes unnecessary energy on the mobile devices.

A number of past studies have reported the significant effect of the tail time on mobile devices' energy by demonstrating that the energy spent during the tail time corresponds to the $60 \%$ of the total energy consumed by the device radio $[5,12]$. These works suggested better utilizing the tail time, ideally by delaying or scheduling the outgoing messages in the device end by modifying the mobile operating systems. In contrast, we approach this problem from the network's perspective and propose a scheduling system for the incoming push notification messages. Our method delays push notification messages and sends them in batches or piggybacked with data traffic in an effort to conserve the energy spent as part of the tail time. More precisely, the system resides on the network operator hub and is capable of sensing and predicting a user's network activities. It intercepts and assign a time-to-live (TTL) to the incoming notification which best corresponds to the users temporal activity pattern, and then uses the TTL information to delay the notifications. Based on a trace based evaluation using cellular network logs of 60 users for 30 days, we shows that it can decrease the energy consumption of mobile devices by $10 \%$ for an average delay of 150 seconds in notification delivery.

The remaining of this paper is structured as follows: we first provide the background context on the cellular network interface, its 
energy model and the existing push notification service architecture in Section 2. We then describe the scheduling system and its components in Section 3. We describe the dataset at hand in Section 4.1, before presenting the results of our evaluation in Section 4. In Section 5, we position our work against the current state-of-the-art that addresses the tail inefficiency. Finally we conclude the paper by discussing the limitations and implications of the proposed system in Section 6.

\section{BACKGROUND}

In this section, we briefly cover the background information regarding i) how resource allocation works on UMTS (Universal Mobile Telecommunication System) networks, and its implications on mobile device energy usage, ii) the architecture explaining how the push notifications currently work and are delivered to users devices.

\subsection{UMTS and its Energy Model}

The UMTS ${ }^{\mathrm{a}}$ network consists of three interacting elements: Core Network (CN), UMTS Terrestrial Radio Access Network (UTRAN), and User Equipment (UE). The UTRAN provides the air interface access method for User Equipment to connect to $\mathrm{CN}$. It consists of two components Node-B (i.e., base stations), and Radio Network Controllers (RNC). RNC is a key element in the UMTS network and is responsible for the radio resource management as well as management of the multiple Node B instances (i.e., Base Stations), to which the UE connects through radio physical channel. The $\mathrm{CN}$ is the backbone of the cellular network and its main functions are to provide switching, routing and transit for user traffic, and to host user database and network management functions. The $\mathrm{CN}$ typically performs these operations via its Gateway GPRS support node (GGSN), responsible for the inter networking between the UMTS network and external packet switched networks, like the Internet and X.25 networks and Serving GPRS support node (SGSN), responsible for the delivery of data packets from and to the mobile stations within its geographical service area.

The power consumption of the UE is influenced by the Radio Resource Control (RRC) states and the Radio Link Control (RLC) protocols. A single RRC state machine is maintained at both the UE and the RNC (i.e., they are always synchronized), and its purpose is to effectively utilize limited radio resources to improve power consumption.

Typically there are three RRC states: IDLE, CELL_DCH, and CELL_FACH as shown in Figure 1. Each of these states are allocated varying radio resources and power.

- The IDLE state happens when there is no network activity on the device, e.g., when a handset is turned on and the RRC connection with RNC is not established. The power consumption of the radio interface in this state is almost zero. Any application traffic triggers a RRC state transition from IDLE state to the CELL_DCH.

- In the CELL_DCH, a RRC connection is established, and a dedicated physical channel is allocated for the UE in both uplink and downlink providing higher data rates.

- In the Forward Access Channel (CELL_FACH) the UE is assigned a default common or shared transport channel in the uplink and monitors the downlink continuously. The UE therefore can transmit small data packets at lower data rates while at CELL_FACH.

\footnotetext{
${ }^{a}$ Although we focus $3 \mathrm{G}$ technology for the work in context, the basic principles of networking remain the same for $4 \mathrm{G} / \mathrm{LTE}$.
}

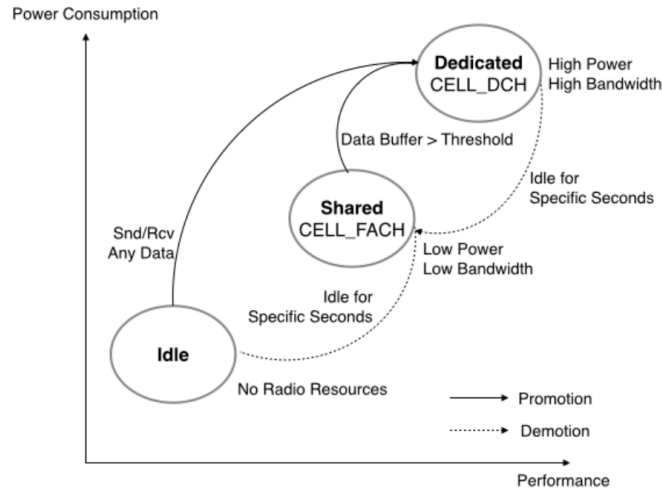

Figure 1: RRC State Machine for UMTS Network

The RRC State transitions between these states are caused by traffic volume and inactivity timers controlled by the RNC. Most of the operators maintain statically set inactivity timers to control the state transitions from CELL_DCH to CELL_FACH as well as from CELL_FACH to IDLE. For example, when a UE is in the CELL_DCH state for a specific time period without any or small data transmission, the RNC releases the dedicated channel and switches the UE state to CELL_FACH. While the UE waits for the inactivity timers to expire, it maintains transmission channels and its radio power consumption is kept at the corresponding level of the state even though it does not send/receive any data. This power inefficient duration is often referred to as tail time. Due to this tail time, transmitting even a small amount of data can cause significant radio resource and power consumption.

\subsection{Mobile Push Notification Service}

Mobile push notification describes a style of internet-based communication where cloud-based applications can send brief alerts and updates to a client application running on a mobile device. The service provides a simple, lightweight mechanism to tell mobile applications to contact the server directly, to fetch the updated application or user data. Mobile operating system providers facilitate this service through dedicated notification servers, e.g., Apple Push Notification Server (APNs), Google Cloud Messaging Server (GCMs), etc. They are also responsible for all aspects of queuing of messages and delivery to the target application running on the target device.

Figure 2 illustrates interactions in the push notification eco-system where the stages are labelled numerically. The process starts with a client application requesting for a device token from the mobile operating system (1), which in turn contacts the respective notification server (2). This device token is then passed to the device operating system (3) and through the client application (4) to the content provider (5), which uses it for pushing subsequent notifications (6). Finally, the notification servers send this notification to the target device (7) by taking into account the notification type, its priority, expiry time and the availability of the device. While stage 1-6 describe an initialization and security handshake between the third content provide and the application, we are interested in stage 7 at which the delivery of the notification to the device occurs. This is done through the persistently maintained connection between the notification server and the mobile device. The typical payload of notification messages are fairly small. In addition, recent notification protocols also provide service providers with op- 


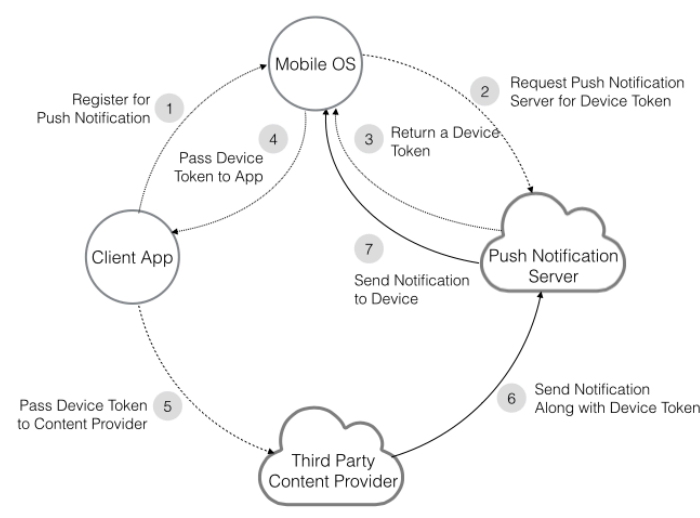

Figure 2: Architecture of Push Notification Service

tions for setting the expiry time and priority of a notification. For example, with APNs a notification message may include an expiration date that identifies when the notification is no longer valid and can be discarded from the APNs queue. If this value is non-zero, APNs stores the notification and tries to deliver the notification at least once within the stipulated time otherwise APNs delivers the notification immediately should the target device be reachable. Furthermore, a notification might include a priority flag that could be set by the content provider to indicate whether or not to delay the notification when the device is idle. For example, Microsoft Push Notifications(MPN) services allow for the content provider to append the NotificationClass in the HTTP header of the notification URI. The notification classes for Windows 8 are defined and fixed to i) Priority indicating the notification is delivered within $450 \mathrm{sec}$ onds; ii) Regular indicating the notification is delivered within 900 seconds; and iii) Real time indicating immediate delivery. These different classes allow the mobile OS and the MPNs to work in conjunction to batch notifications so as to save energy. Although, the current extensions allow for some level of control over the delivery of the push notifications, the delaying period defined by the push notification servers (e.g., MPNs) are arbitrary and not reflective of the device's RRC state.

\subsection{Device-Centric Notification Management}

Notification management on the device-end can be done either through applications or the OS itself. From the OS aspect, iOS, Android and Windows OS all provide the user with settings that allow them to indicate the willingness to receive notifications from their installed applications. These settings are limited to true/false with no personalization regarding the sensitivity and priority of the notifications from different applications. With regards to applications, various notification management applications are designed for Android OS. They mostly allow the user to classify notifications and organize them based on their preferences. While most of these applications provide an alternative interface to the notification center on Android devices, some also enable the user to ignore notifications from unwanted contacts or applications. However, any filtering on the notification is done at the device-end and requires the notification to be delivered to the device at the first place.

Alternatively, recipe based approaches, such as IFTTT (IF THIS THEN THAT), allow the user to set specific rules regarding their content from various channels (e.g., Facebook etc.) and the actions that are to be applied once a condition is met. Using this mechanism the user can create a more personalized notification delivery (e.g., when tagged in Facebook), enabling the IFTTT to pull content when the specified rule occurs. The IFTTT application persists a connection with the content provider channel through a polling period. However, in order to work as notification delivery, the polling period would need to be set to a very short time period (almost continuously). Thus making such application based approaches even more energy consuming than the traditional push notification delivery.

\subsection{Push Notification Scheduler: A Remedy}

Although, the notification servers offer some controls to content providers to qualify a notification in terms of its priority and delay tolerable property, they are not aware of the radio states of the target devices. Hence, high frequency of the notifications could increase the time during which the device ratio spends in tail time needlessly and thus draining significant amount of battery. In addition, the sporadic nature of the notifications also contributes to significant energy drain. This is because the majority of the network operators' dormant timer $^{\mathrm{b}}$ is set to 30 seconds or less [2], thus making the delivery of sporadic notifications even more energy consuming. As Balasubramanian et al. have shown in [5], 60\% of the total energy consumed for data communication corresponds to the tail energy, and the high frequency and sporadic bursts of notifications only adds to this drain. This is a remarkable overhead considering the past research has shown not all the notifications are perceived as important to the user and many of the notifications are ignored [13].

The work presented in this paper primarily aims to address this problem by delaying the notifications and piggybacking them with larger data traffic when possible. To accomplish this, our system is placed at the mobile operator end for accurate awareness of the radio states of the devices, and leverages a network traffic predicator to determine the appropriate delay interval for the notifications.

Such a system is made possible with the advent of the Network Function Virtualization (NFV), where the network components are no longer provided in closed "black-box" but are placed in virtualized servers [1]. This paradigm facilitates the network operators to offer new services to their users at little cost without complex modifications in the network components and any concerns about scalability of the component. Similarly in this paper, we exploit such underlying functionalities of NFV to design a push notification scheduler.

\subsection{Cellular vs WiFi}

Service providers take different approaches for delivering the notifications with respect to the access technology. Android devices for example always use the default interface to access GCM servers. iOS devices on the other hand use their cellular interfaces to connect to APN servers even if they are connected to a WiFi network. They fall back to WiFi only if the cellular connection is not available. When the push notifications follow different paths than regular data traffic, it is not possible to piggyback the notification messages to non-notification packets. In addition, it has also been shown that the tail energy is not a grave problem with WiFi [10]. As a result, extending the notification scheduler to WiFi networks does not add substantial value, and our system is exclusive for devices that are only connected to a cellular network.

\section{SYSTEM DESCRIPTION}

In this section, we discuss the basic working principles of the notification scheduler. We propose to extend the basic functionality of the core network element of UMTS network by adding push notification schedules as a component. We place the scheduler on

\footnotetext{
${ }^{\mathrm{b}}$ The time based on which RNC releases high power state channels
} 
the data plane of the cellular core network instead of the RNC so that it can sense when the device has any incoming or outgoing traffic, essentially to be aware of the radio state of device even though the actual state machine interface is not maintained there. It can be co-located with either the Serving GPRS Support Node (SGSN) or the Gateway GPRS Support Node (GGSN), due to their functions on the data plane. The scheduler is designed as a modular system composed of three elements, and leverages the Deep Packet Inspection (DPI) capabilities available at the core network: These elements are:

1. Burst Detector: This element is responsible for capturing a group of traffic transactions (upload or download) that constitute a single burst of network activity.

2. Prediction Model : This element uses the traffic burst pattern to maintain a model of a user's network activity, based on which it can provide an estimated delay that a notification can tolerate for that specific user.

3. Notification Scheduler: This element is responsible detecting the push notification messages through TCP port monitoring and leverages the prediction model to determine the delay interval for the notification and accordingly schedule the delivery of the notification.

\subsection{Burst Detector}

A traffic burst represents a group of transactions under one single data transmission session. Typically a User Data Record (UDR) entry for a data transfer contains user identity as International Mobile Subscriber Identity (IMSI), transaction time, the amount of data being exchanged in the transaction, Node-B information, host information, etc. To determine a network activity session, it is necessary to identify and group a set of UDR entries together that appear in close temporal proximity and are separated by a specific time interval from the subsequent group of UDR entries. These groups of UDR entries or traffic bursts then can be ordered by time to construct a network activity trajectory, which can later be used for modeling temporal behavior of an individual. The Burst Detector maintains a temporary storage for each individual IMSI. As UDR entries come in for each IMSI, the Burst Detector checks the timestamp of the latest entry and compares it with the most recent entry to determine the arrival interval. If the interval is longer than a selected threshold, then the set of entries that are in the storage are grouped as one burst and is passed to the Prediction Model as an identified traffic burst. Otherwise the entry is added to the storage as a constituent of ongoing burst. For the dataset presented in this paper and discussed in section 4.1, the interval threshold is set to 31 seconds which was selected empirically to minimize isolated transactions to appear as one small burst.

\subsection{Prediction Model for TTL Assignment}

In order to ensure that our system accounts for users individuality and their different behavioral patterns, we require an adaptive mechanism which would assign a time-to-live (TTL) to each notification by evaluating its priority to the end user. It is possible to model this priority in two different ways. A content-centric approach in which the priority is decided based on the nature of the content and its sensitivity to delay. For example, a location sharing application may not tolerate any delay in its notification and thus require the TTL to be set to zero. It is worth noting that the Notification Servers such as APNs and GCM already account for this simple feature by allowing the application developers to set a priority field. Alternatively the priority can be set based on the

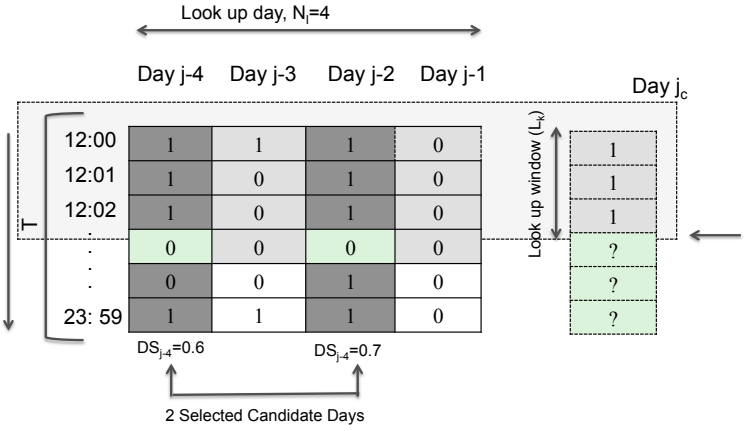

Figure 3: Example of the Network Activity Prediction and TTL Estimation Process

popularity of the content and based on how it would be perceived by the user. A user-centric approach on the other hand accounts for the sensitivity of the user to the delay at a given time. In other words, the likelihood that the user would indeed attend the notification. Mashhadi et al. [13] has previously shown that the likelihood of a user attending a notification is much higher when the user is already engage with their phone. Similarly we take into account, user's engagement in an online activity on their device to model the delivery time of the notifications (i.e., TTL).

We design an algorithm that leverages the temporal activity ${ }^{c}$ behavior of the user to estimate the likelihood of the user interacting with their mobile device at a given time. Using this adaptive mechanism, we are able to predict the earliest time that the user will start a data session and thus assign the TTL accordingly. Although we grant a user-centric approach for our scheduling approach, it is worth noting that the two approaches of content and user-centric are not exclusive and could be combined in the future systems.

Our algorithm predicts upcoming network activity (i.e., next traffic burst) of current day by matching patterns of similar days in the past. We build a $T \times N_{l}$ matrix $U$, where $T$ denotes the number of time slots in a day and $N_{l}$ is the number of look up days. Each element $u_{i j}$ in $U$ is a binary value that represents whether a user's was engaged in any network activity at the time slot $i$ of the day $j$ ( $i \in T$ and $j \in N_{l}$ ). Our algorithm uses $U$ to predict whether a network activity is likely to occur in the next prediction horizon window (denoted as $f$ ) of the current day by matching patterns of similar days in the past $N_{l}$ days. The current day and the current time slot are denoted as $j_{c}$ and $i_{c}$, respectively (i.e., the current network activity value is $u_{i_{c} j_{c}}$ ). As a day progresses, network activity vector is constructed from midnight up to the current time. To predict the next upcoming networking activity upon the arrival of a notification at time $t_{n o t f}=i_{c}$, we set a look up window of $k$ immediate past slots denoted as $L_{k}$ and run a similarity measure that compares the network activity of past $L_{k}$ slots of the current with the corresponding time slots of previous $N_{l}$ days. $M$ top most similar days are selected where $M<N_{l}$.

To obtain a similarity value of the current day to the past days we compute a binary similarity measure [6]. We have examined several binary similarity measures with our dataset by dividing our samples into subsets randomly. We have found that Sokal-Michener measure offers the best discrimination capability [19]. Therefore, to compare the similarity between two temporal activity vectors $x$ and $y$ with length $k$ representing network activity of past $L_{k}$ slots

\footnotetext{
${ }^{\mathrm{c}}$ By activity we refer to users activity on the device which generates data traffic
} 
of today and one of the past $N_{l}$ days respectively, we define a temporal day similarity score for each of the $j^{\text {th }}$ day as:

$$
D S_{j}=\frac{1}{k} \sum_{s=1}^{k} I\left(x_{s}^{t} y_{s} \| \bar{x}_{s}^{t} \bar{y}_{s}\right)
$$

where $I(r)$ is the indicator function, and $I(r)=1$ if $r$ is true or 0 otherwise, $x_{s}^{t} y_{s}$ denotes the positive match and $\bar{x}_{s}^{t} \bar{y}_{s}$ denotes the negative match at $k^{t h}$ position between $x$ and $y$,

Once the day similarity scores are obtained, the algorithm moves to the selection of the $M$ candidate days. This selection is performed by sorting $N_{l}$ past days twice, first on the day similarity score and sorting on the time difference from the current day. It is worth noting that in cases where there are no similar days $\left(D S_{j}=0\right)$, the algorithm will pick the candidate days based on the most recent ones. Finally, the prediction algorithm considers the network activity vector of each candidate day for the upcoming slots (prediction horizon window $f$ ) and computes the probability of occurrence of a network activity, e.g., presence of network traffic for each of the upcoming slots. If the probability is higher than a selection threshold $p_{t h}$ then that network activity in the prediction slot is set to 1 or 0 otherwise. After ROC analysis, we set $p_{t h}$ to 0.6. The algorithm performs this step by taking each slot at a time and combining day similarity score to ensure that most similar and more recent days have highest contribution in predicting the occurrence of a network activity. The $t t l$ is then set to the first time slot which is predicted to have an activity $\left(u_{i_{f} j_{c}}=1\right)$.

$u_{i_{f} j_{c}}= \begin{cases}1 & \text { if }\left(\sum_{j=1}^{M} D S_{j}\right)^{-1} \sum_{j=1}^{M} D S_{j} I\left(u_{i_{f} j}=1\right)>p_{t h} \\ 0 & \text { otherwise }\end{cases}$

When the prediction slot $i_{f}$ is at the beginning or end of a day, i.e. before midnight or just after midnight, the lookup slots for similarity matching are determined from the immediate previous day, and candidate slots for prediction are selected from the immediate next day. This avoids complexities in making predictions that span midnight. Algorithm 1 summarizes the described prediction method.

Figure 3 illustrates a simplified example, where the number of look up days $N_{l}$ are set to 4 and the temporal granularity is in minutes that is $T=1440$ (matrix $U$ is $1440 \times 4$ ). In this example a notification has arrived at time $t_{n o t f}=12: 03$, and we want to estimate the next upcoming slot $i$ where there would be any networking activity. The algorithm starts with detecting the similar past days to the current day $j_{c}$ from the look up window $N_{l}$, where 2 days are selected. We then compute by considering the candidate days $(j-4$ and $j-2)$ the likelihood for the activity at $i_{c}+2$ would be 1 with $p_{t h}=0.6$. The algorithm stops when it finds a candidate time slot (i.e., of predicted value 1) and would return $t t l$ as 2 minutes, i.e., 120 seconds.

\subsection{Notification Scheduler}

The notification scheduler has the ability to detect if a packet is a notification and can intercept traffic. The push notifications on mobile device typically use a particular port number while connecting to the push notification servers. For example, Android devices use TCP port 5228 to connect to GCM Servers. iOS devices on the other hand use TCP port 5223 to connect to the APN servers. Though the devices fall back to other ports in case they cannot establish connection on these ports (e.g., TCP port 443 for Android and iOS), it is safe to assume these ports are open on a cellular network. Hence, notifications can be detected by looking at the protocol field in the IP header to check whether TCP is used

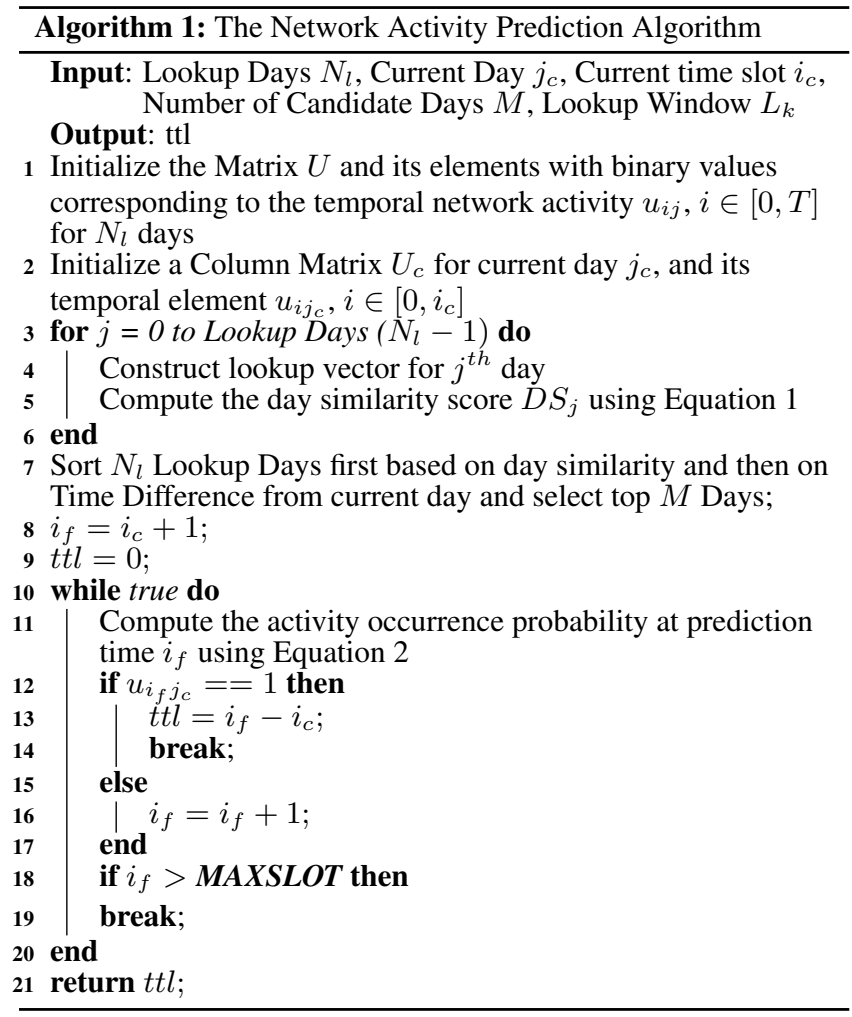

and which destination port is included in the TCP header. Traffic that corresponds to the data messages rather than isolated notification messages are sent immediately to the users by the notification scheduler. The notification messages on the other hand are delayed to conserve energy on the devices. The decisions to delay the notifications are governed by the outcome of the prediction model.

Recall from the previous section that each notification is associated with a TTL by which the messages needs to be sent. In accounting for TTL, the scheduler adds an expiration timer to the queue that holds all the notification messages destined for a device, that is set to the minimum TTL among the messages in the queue. The scheduler sends all the notifications to the destination when the timer expires. If a data message that cannot be delayed arrives before the timer expires, the scheduler piggybacks the notification messages to the data and cancels the existing timer.

\section{EVALUATION}

In this section, we first discuss the dataset used in our analysis. We then describe the metrics and benchmark used in our evaluation. Then, we discuss the performance of the prediction algorithm and finally, we report on the results of out scheduler in terms of energy gain and delay in comparison with the baseline approaches.

\subsection{Datasets}

We obtained a dataset of anonymized network activities of 60 users for over a month period in March $2013^{\mathrm{d}}$.This data comprises the size (both uplink and downlink), time and the duration of all incoming and outgoing network traffic for each user. In order to preserve the privacy of the users, the users are presented by random ids

\footnotetext{
${ }^{\mathrm{d}}$ Specific details about the identity of the network provider, as well as the location and time of the UDR data are omitted in order to preserve the anonymity of the network operator.
} 


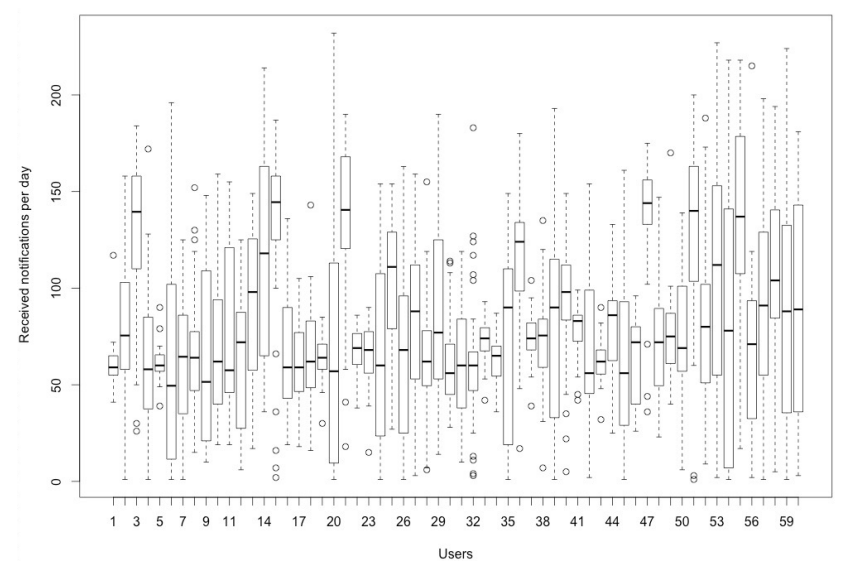

Figure 4: Number of Notifications per User per Day.

and their phone number is dropped from the database. Additionally any information regarding the location (e.g. the cell tower location, GPS etc.) has been also removed from the dataset. The data at hand is restricted in two aspects, firstly it does not contain any host URL information, making it impossible to determine whether a packet was sent by a push notification server; secondly it does not include the destination port number, thus making it further difficult to detect push notifications by monitoring the traffic to the specific port (e.g., port 5028 for Android devices and 5223 for iOS).

In order to identify the push notifications for the purpose of testing our scheduling algorithm, we require an alternative way for identifying the notification messages. To do so, we assume that the download traffic packets which are small in size and has occurred in isolation, that is they are neither triggered or followed by any upload requests from the device, correspond the push notifications. In other words, the traffic that has occurred at least $t_{\text {tail }}$ seconds after its preceding traffic and is not followed by any other traffic for at least $t_{\text {tail }}$ seconds; where $t_{\text {tail }}$ is the tail time parameter. We set the size for the determined notifications to less than 300 bytes as it corresponds to the iOS notifications defined by APNs (256 bytes) at the time data is collected. We set $t_{\text {tail }}$ to 30 seconds as it is commonly used by the network operators.

Although we cannot ensure that all the detected small isolated traffic from our dataset are indeed the push notification messages (we may have false positive), we can argue that all the push notifications in our dataset are detected (no true negative). In other words, alongside those detected push notifications we may also observe other packets that are small isolated download traffic such as those packets corresponding to advertisements. We argue that should these false positive cases happen to be part of a larger traffic, they would be have been followed by immediate data transaction either before or after them. However, as they are detected in isolation, they correspond to the cases where the data transmission would have caused the cellular interface to stay unnecessary in the tail state with no upcoming traffic.

The resulting dataset consists of 143,261 isolated notifications and 765,739 traffic bursts. Figure 4 further illustrates the number of detected notifications for each user per day. On average we find that the users received around 80 notifications per day (median=74). However, as seen from Figure 4, some users receive as many as 200 notifications on some days and only a few on other days. This trend corresponds in size and distribution to those push notifications collected by Pielot et al [15] and also those observed

\begin{tabular}{l|c}
\hline Parameter & Value \\
\hline DCH tail power base & $803.9 \mathrm{~mW}$ \\
DCH tail duration & $8088.2 \mathrm{~ms}$ \\
FACH tail power base & $601.3 \mathrm{~mW}$ \\
FACH tail duration & $824.2 \mathrm{~ms}$ \\
Transmission Power & $991.67 \mathrm{~mW}$ \\
Uplink Bandwidth & $200 \mathrm{kbps}$ \\
Reception Power & $939.89 \mathrm{~mW}$ \\
Downlink Bandwidth & $1 \mathrm{Mbps}$ \\
\hline
\end{tabular}

Table 1: Power Parameters for 3G Radio State Machine

by Mashhadi et al. [13] from empirical study of notifications on the mobile devices.

\subsection{Metrics, Benchmark and Simulation Setup}

For evaluation of the notification scheduler, we selected two metrics: delay, that is how long on average (in seconds) notifications were delayed by our scheduler, and energy that is how much energy (in Joules) our scheduler saved. Finally, we evaluate and discuss the trade-off between these two metrics for our scheduler.

In order to measure the energy gain and delay imposed on the notifications, we use a baseline scheduling approach. For this purpose, we choose the state-of-the-art notification delivery in the network that immediately sends the notification to the destination device. We refer to this approach as, Send Immediately. This approach serves as a baseline for delivery time, allowing us to calculate the delay introduced by our scheduler. We also select a second baseline approach which corresponds to minimum energy consumption, albeit conveying maximum delay. In this approach all the notifications are delayed and piggybacked with the next data traffic. We refer to this baseline as Send with Next Non Notification Data. Finally, we also report the performance of our scheduling method for two variant settings of the TTL : fixed, where we set the TTL to a fixed value, and adaptive where the TTLis set based on the output of the traffic prediction.

We use a server-client model to simulate the cellular network. In this setup, there exists a number of clients and each corresponds to a base station. The client hosts a number of threads each modeling the radio state machine of a user. At the end of a simulation run, each thread yields the cumulative energy consumption of the corresponding user's cellular radio in 15 days. This value does not include other factors that contribute to the energy consumption of the device such as display, processing, etc. The state machine transitions are triggered by the arrival or transmission of data messages and the expiration of inactivity timers as explained in Section 2.1. The power consumption levels in each state and the timer expiration duration values are adopted from [10]. Relevant parameters are summarized in Table $1^{\mathrm{e}}$.

In our trace driven simulations, the first 15 days are used to train the prediction algorithm. The second 15 days are used for evaluation. The downlink traffic in this trace yields the data sent from the server to the client. The client then pushes it to the thread that corresponds to the user radio state machine. The uplink traffic on the other hand is originated by the user module and pushed to the client and then to the server. The server acts as the location where notification scheduler is placed that forwards the packets to the their

\footnotetext{
${ }^{\mathrm{e}}$ We neglect the power associated with the radio state promotion.
} 


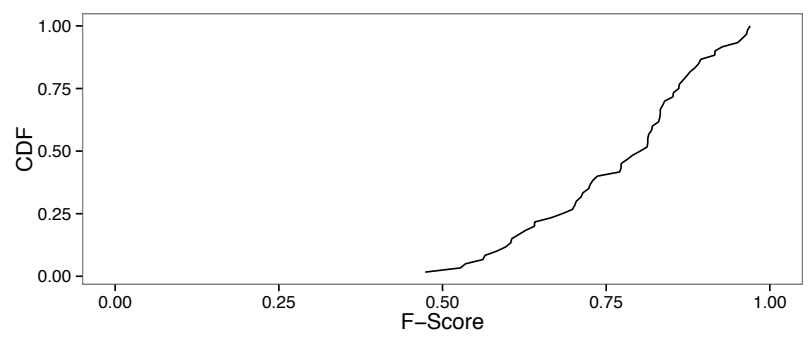

Figure 5: Cumulative Distribution of Prediction Performance over all 60 Users

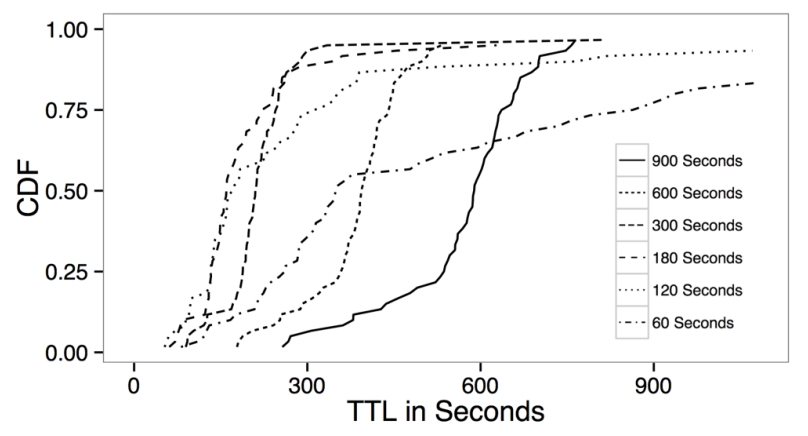

Figure 6: Influence of Varying Slot Duration (in seconds) on the TTL Estimation

destinations. The server also determines how long the notifications are suspended using the baselines or our mechanism.

\subsection{Performance of Prediction Algorithm}

Predicting a network activity for a future hour slot is essentially a classification problem and the performance of the algorithm can be evaluated by standard Information Retrieval measures. For each time slot $i_{f}$, let $T$ be the true network activity, and $S$ be the predicted set activity. Accuracy is measured by the Hamming Score which symmetrically measures how close $T$ is to $S$, i.e., Accuracy $=$ $\frac{\|T \cap S\|}{\|T \cup S\|}$. Precision $(P)$, Recall $(R)$ and F-Measure $\left(F_{1}\right)$ are defined as $P=\frac{\|T \cap S\|}{\|S\|}, R=\frac{\|T \cap S\|}{\|T\|}$ and $F_{1}=\frac{2 P\left(i_{f}\right) R\left(i_{f}\right)}{P\left(i_{f}\right)+R\left(i_{f}\right)}$.

For evaluating the algorithm, we split 30 days of data into two parts. The data of first 15 days are used to train the algorithm, e.g., as histories of network activities and the data of remaining 15 days are used to evaluate the performance of the algorithm.

Figure 5 plots the cumulative distribution of F-Score across all 60 users with 180 seconds as temporal slot duration, 3 hours as lookup slot duration, 15 look up days, 5 candidate days, and 0.6 as the selection threshold. As we observe, at least $60 \%$ of the users have over $0.7 \mathrm{~F}-\mathrm{Score}$, which is considered reasonably high. Prediction performance remains consistent with varying lookup and candidate days. Figure 6 illustrates the impact of slot duration on the TTL assignments (in seconds). We observe that larger temporal slots increase the TTL duration. Based on these observations, in the rest of this section, we evaluate the performance of notification scheduler through a simulation model in which we construct the prediction model with the parameter as depicted in table 2 .

\subsection{Performance of Notification Scheduler}

We first start by measuring the energy gain of our approach for all the users. Figure 7, illustrates the extent of energy consump-

\begin{tabular}{l|c}
\hline Parameter & Value \\
\hline Lookup Days & 15 \\
Candidate Days & 5 \\
Slot Duration & 180 Seconds \\
Lookup Slot & 3 Hours \\
Selection Threshold & 0.6 \\
\hline
\end{tabular}

Table 2: Prediction Parameter for the Simulation Model

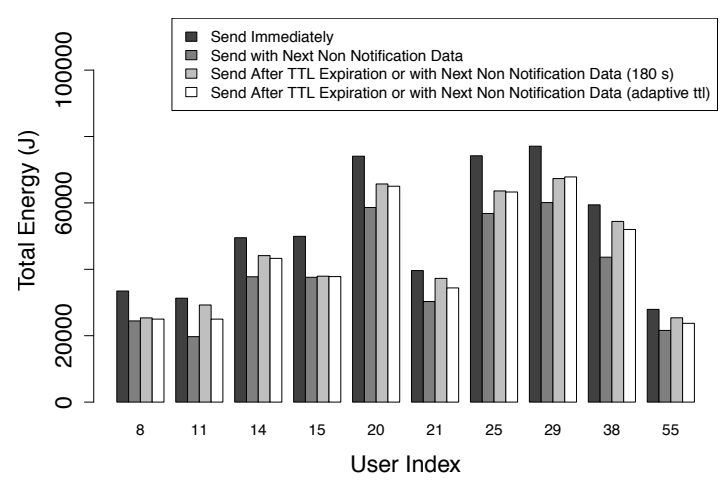

Figure 7: Energy Consumption for Each User with Scheduling Conditions

tion of our scheduler comparing to the baselines for 10 selected users. Even though, we only show the energy consumption for 10 users for the clarity in the presentation, the observation is consistent across all users. The figure also illustrates the inefficiency of the current notification delivery approach (i.e.,Send immediately). Looking deeper at the results, we also observe that our second baseline that is suspending the delivery of the notification until the Next non-notification data transfer, can on average conserve $15 \%$ energy $(\sigma=0.06)$. These results suggest the maximum upper bound that is possible in energy saving at the cost of maximum delay.

Next, we evaluate our scheduling mechanism (i.e. Send After TTL Expiration or with Next Non Notification Data) both with a fixed TTL and an adaptive TTL for each notification using the prediction algorithm. In both cases the notification is sent with the upcoming traffic or if the TTL reaches. We evaluate by setting the fixed TTL value for this heuristic to 180 seconds. Comparing the two, we can observe from the Figure 7 (and for the all the 60 users) that with the adaptive TTL the energy consumption is lower than the fixed TTL. A pairwise t-test reports of this consistent observation across all users with the mean difference of 621 Joules $(t=6.16, p<0.0001, d f=59)$.

Figure 8 shows the amount of energy consumption by all the users in the span of 15 days. Send with Next Non Notification Data saves $16 \%$ energy in comparison to Send Immediately. The energy savings for Send After TTL Expiration or with Next Non Notification Data with adaptive TTL amounts to $11 \%$ energy savings. Even with a TTL that is as low as 180 seconds, the mechanism save $10 \%$ energy.

In Figure 9, we show how the total energy consumption across all the users changes when Send After TTL Expiration or with Next Non Notification Data uses various fixed TTL values. Note that 


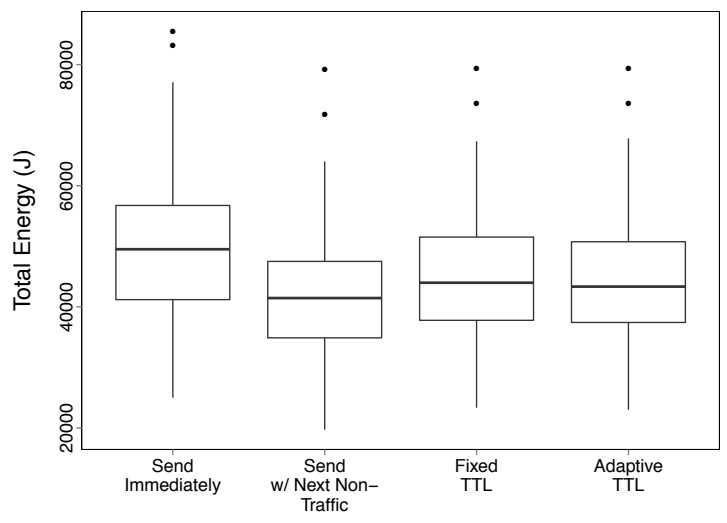

Figure 8: Energy Consumption vs Scheduling Heuristic

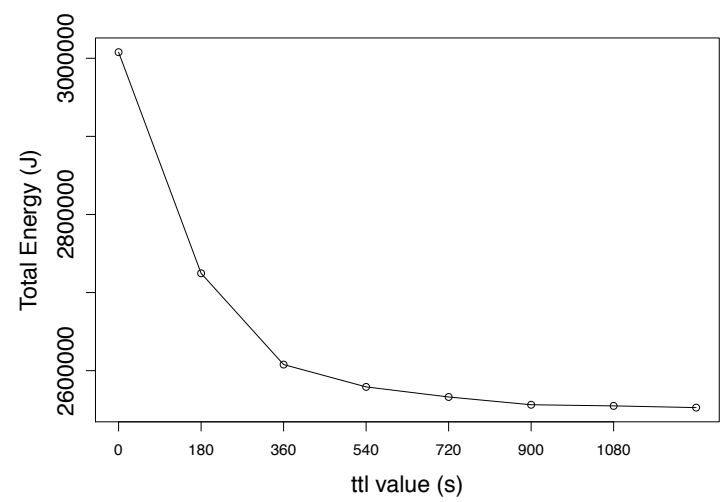

Figure 9: TTL value vs Energy

TTL $=0$ corresponds to Send Immediately, and the last data point corresponds to Send with Next Non Notification Data baseline, which gives the lower bound in energy consumption. We see that as the TTL increases, the resulting energy consumption approaches the lower bound. When the TTL=18 minutes (1080 seconds), the energy consumption is only about $1 \%$ more than the lower bound.

Figure 10 shows the latency caused by the notification scheduling mechanisms for the set of selected 10 users. A t-test analysis indicates the significant difference between the adaptive and fixed TTL approach for all the users $(t=-4.9848, p<0.0001$, $d f=59)$. Since Send Immediately immediately sends the notifications without intercepting them, it does not cause any latency. As expected the Send with Next Non Notification Data suspends the notifications for the longest duration, providing an upper bound to the delay. These findings are parallel with the energy observations in Figure 7. To present this trade-off the overall latency introduced by the scheduler is reported in Table 3 along with the average percentage of energy saving. These results are encouraging as they confirm the performance of our scheduler. That is although Send After TTL Expiration or with Next Non Notification with adaptive TTL consumes only 5\% more energy than the lower bound in Send with Next Non Notification Data, it takes 32\% less time to deliver notifications, resulting in on average over 2 minutes delay only.

Also note that in this set of simulations, the tail time we use is around 9 seconds which is adopted from [10]. Tail time values are set by the operators and can be as high as 30 seconds. The benefits

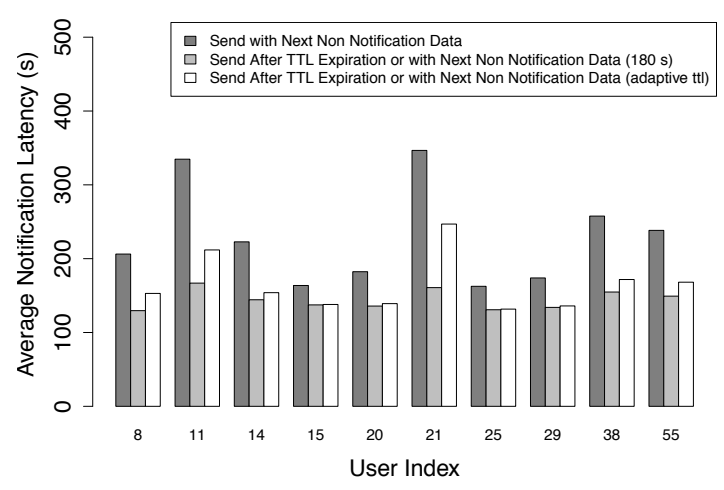

Figure 10: Latency of the Notification Selivery for Each User

\begin{tabular}{l|c|c}
\hline $\begin{array}{l}\text { Scheduling } \\
\text { Condition }\end{array}$ & $\begin{array}{r}\text { Average } \\
\text { Latency } \\
\text { (in Sec) }\end{array}$ & $\begin{array}{c}\text { Average } \\
\text { Energy } \\
\text { Savings }\end{array}$ \\
\hline $\begin{array}{l}\text { Send with Next Non } \\
\text { Notification Data }\end{array}$ & 230 & $15 \%$ \\
\hline $\begin{array}{l}\text { Send After TTL Expiration } \\
\text { or with Next Non Notification } \\
\text { Data (TTL = 180 s) }\end{array}$ & 136 & $9 \%$ \\
\hline $\begin{array}{l}\text { Send After TTL Expiration } \\
\text { or with Next Non Notification } \\
\text { Data (adaptive TTL) }\end{array}$ & 157 & $10 \%$ \\
\hline
\end{tabular}

Table 3: Latency versus Energy Saving with the Scheduling Conditions

of our approach would have been even higher with higher tail time values.

\subsection{Limitation}

In this paper we have designed a system for scheduling the notifications and where possible we have adhered to a generic solution. However, due to the limitations imposed by the data at hand, we had to apply some restrictions to the evaluation of the proposed system.

We granted a trace-driven analysis in order to evaluate the energy savings on the end-user devices. We understand that as the result of this approach, we are indeed facing a "Butterfly Effect" where tempering with an initial condition (i.e., delaying a notification) may result in a different course of future events (e.g., delayed communication) than what we originally have in the traces. Although, the course of future data traffic that is exhibited may be different, we believe our main findings regarding the quantification of the energy saving stays valid. The values reported in this section are not meant to be taken as absolute values but rather as bounds in the extent of energy savings.

In selecting the required parameters for our system, we had to adhere to an assumption that all the notifications are issued by APNs and are intended for iOS devices, that is they are less than 300bytes in size. We adhered to this choice as our data does not include information regarding the individual devices or port numbers. Fur- 
thermore, the alternative thresholding for Android notifications is indeed much bigger in size which would have result in the detection of many more notifications and a better performance of our system.

\section{RELATED WORK}

In [5], authors show that the energy consumed in the tail state corresponds to the $60 \%$ of the total energy consumed for data communications. They propose an algorithm that schedules and aggregates the outgoing small transmissions into large ones so that the occurrence of tails (and thus energy consumption) can be reduced. In [8], the authors use machine learning techniques to predict the network activity on the phone, which is used to manipulate the state transitions on the phone. Another relevant technique is TailTheft [12], which uses the duration of tail state to prefetch content and deferred messages for delay tolerant applications. Sending location update messages in an energy efficient way is addressed in [4]. In this work, messages to the location servers may be delayed as long as some quality measures such as time elapsed since the last message, the distance from the last reported location etc. are satisfied. In all these works, the traffic scheduling is considered only for outgoing packets as the scheduler runs on the phone, and requires modifications on the operating system or on the apps as well as resources to perform these computations. In contrast, our method requires no changes to the mobile phone and runs in the network.

A broad body of research addresses tail tuning and termination. In [7] and [9], different values for tail time has been suggested and proven to reduce the energy consumption. In $[11,17,18]$ dynamic assignment of tail time based on usage pattern has been proposed. In particular [18] relies on prediction and dynamically terminates the tail time when it does not foresee an upcoming activity. Similarly [3] proposes data mining approaches to detect end of communication spurts to invoke fast dormancy with higher accuracy. Finally $[14,16,20]$ focus on modeling and profiling the resource usage on mobile devices. In [16] the authors introduce ARO (Application Resource Optimization) tool that helps the developers to discover the inefficient resource usage by considering a cross-layer interaction for layers ranging from cellular interface to application layer.

\section{CONCLUDING REMARKS}

In this paper we have designed a system for scheduling the push notifications that resides in the core network of a cellular network and is added to the data plane. Our system leverages the paradigm of Network Function Virtualization (NFV). With this paradigm, network components are provided in software in virtualized servers and not in dedicated and specialized hardware platforms. In this way, our scheduler can scale up and down by simply initiating or shutting down virtual machines in which the scheduler runs. The proposed system suspends the notification messages in the network and leverages a DPI that monitors and exploits its past activity to predict when she will be engaged with her device in the near future. This information is then used to schedule the queued notifications. Our results show that in a UMTS network setting, by scheduling notifications it is possible to save $11 \%$ in average.

Although, we presented our approach for the UMTS setting due to the dataset at hand (extracted from UMTS network), our system can easily be adapted to the younger generation networks such as LTE. In a UMTS network, the scheduler can be situated along with Serving GPRS Support Node (SGSN) or Gateway GPRS Support Node (GGSN). In LTE evolved packet core (ePC), the scheduler may be situated with data plane elements Service Gateway (SGW) and the PDN gateway (PGW). SGW is a better option as the location of the scheduling module a mobile device may use more than one PGWs but is connected to a single SGW instance. This way, the scheduler has access to a user's all traffic activity. Due to the nature of DRX (Discontinuous Reception) mechanism which involves a state machine similar to the one used by UMTS, we believe the savings could be more significant with LTE networks. As the previous work has shown that the effect of the tail energy is even more drastic in LTE networks than 3G networks [10].

Another aspect of our implementation is that an instance of components like GGSN and SGW only serve a particular geographical area. We do not introduce any handoff mechanism for users moving from one area to another area. Rather, we assume the notification scheduler in an area only stores and uses data regarding user behavior in that geographical region. In so doing, we are implicitly introducing a spatial element to the notification scheduler. As the by product of this design decision, our activity prediction could be extended to take into account the spatial features (e.g., activity in significant places such as home) in addition to the current temporal ones. Since the scheduler is implemented in virtualized servers, the system does not need to concern with the inter radio access technology handovers either. The same servers can be utilized with UMTS and LTE network in areas where both services are available.

Moreover, our evaluation was performed on a dataset that extracted from a core network in early 2013. Since then, mobile operating system vendors allow for a larger payload in the notification messages sent by the application providers. In 2013, an APNs notification could fit in a single network packet. However, the payload size can now be as much as 4 Kbytes. With a typical MTU size of 1500 bytes, this can fit in at most four packets considering the lower layer networking overhead. This is still not a large stream and with the state-of-the-art optimizations in TCP, all packets are sent at once without waiting for the acknowledgement for the first packet. Therefore, our approach would work on the current implementation of push notification servers without a need to make any modifications to our system to address such payload sizes.

Finally, this work has important implications regarding conserving tail time energy on end-users device. As our findings have demonstrated, in many cases isolated notifications are pushed by the notification servers such as APNs causing an inefficient establishment of the cellular interface on the device. While the impact of this problem, might not be as exhaustive on today's smartphones where the predominantly used lithium-ion batteries have sufficiently hight capacity, it is to a much greater extent on wearables and other devices where the energy resources are scarce due to form factor ${ }^{f}$. Although, this problem could also be addressed by including time-to-live and priority of the notifications at the content provider side while leveraging an activity prediction from the device $O S$, such approach would not be feasible for devices with limited processing powers. Therefore, we strongly believe the answer in addressing this challenge lies within the network's end as they have a bigger picture of users activities and devices' cellular interfaces, without the need to rely on the device to perform any calculations or communications.

\section{REFERENCES}

[1] Network Functions Virtualisation, An Introduction, Benefits, Enablers, Challenges \& Call for Action. White Paper, SDN and OpenFlow World Congress, Oct 2012.

[2] G. Association. Fast dormancy best practices, version 1.0, 2011.

\footnotetext{
${ }_{\mathrm{f}} \mathrm{http} / / / \mathrm{www}$.newelectronics.co.uk/electronics-blogs/powering-
} wearables-and-giving-batteries-a-better-life/64664/ 
[3] P. K. Athivarapu, R. Bhagwan, S. Guha, V. Navda, R. Ramjee, D. Arora, V. N. Padmanabhan, and G. Varghese. Radiojockey: Mining program execution to optimize cellular radio usage. In Proceedings of the 18th Annual International Conference on Mobile Computing and Networking, Mobicom '12, pages 101-112, 2012.

[4] P. Baier, F. Dürr, and K. Rothermel. Opportunistic position update protocols for mobile devices. In Proceedings of the 2013 ACM international joint conference on Pervasive and ubiquitous computing, pages 787-796. ACM, 2013.

[5] N. Balasubramanian, A. Balasubramanian, and A. Venkataramani. Energy consumption in mobile phones: a measurement study and implications for network applications. In Proceedings of the 9th ACM SIGCOMM conference on Internet measurement conference, pages 280-293. ACM, 2009.

[6] S. Choi, S. Cha, and C. C. Tappert. A Survey of Binary Similarity and Distance Measures. Journal of Systemics, Cybernetics and Informatics, 8(1):43-48, 2010.

[7] M. Chuah, W. Luo, and X. Zhang. Impacts of inactivity timer values on umts system capacity. In Wireless Communications and Networking Conference, 2002. WCNC2002. 2002 IEEE, volume 2, pages 897-903. IEEE, 2002.

[8] S. Deng and H. Balakrishnan. Traffic-aware techniques to reduce $3 \mathrm{~g} / \mathrm{lte}$ wireless energy consumption. In Proceedings of the 8th international conference on Emerging networking experiments and technologies, pages 181-192. ACM, 2012.

[9] H. Falaki, D. Lymberopoulos, R. Mahajan, S. Kandula, and D. Estrin. A first look at traffic on smartphones. In Proceedings of the 10th ACM SIGCOMM conference on Internet measurement, pages 281-287. ACM, 2010.

[10] J. Huang, F. Qian, A. Gerber, Z. M. Mao, S. Sen, and O. Spatscheck. A close examination of performance and power characteristics of $4 \mathrm{~g}$ lte networks. In Proceedings of the 10th International Conference on Mobile Systems, Applications, and Services, MobiSys '12, pages 225-238, 2012.

[11] C.-C. Lee, J.-H. Yeh, and J.-C. Chen. Impact of inactivity timer on energy consumption in wcdma and cdma2000. In Wireless Telecommunications Symposium, 2004, pages 15-24. IEEE, 2004.
[12] H. Liu, Y. Zhang, and Y. Zhou. Tailtheft: Leveraging the wasted time for saving energy in cellular communications. In Proceedings of the sixth international workshop on MobiArch, pages 31-36. ACM, 2011.

[13] A. Mashhadi, A. Mathur, and F. Kawsar. The myth of subtle notifications. In Proceedings of the 2014 ACM International Joint Conference on Pervasive and Ubiquitous Computing: Adjunct Publication, pages 111-114. ACM, 2014.

[14] A. Pathak, Y. C. Hu, and M. Zhang. Where is the energy spent inside my app?: fine grained energy accounting on smartphones with eprof. In Proceedings of the 7th ACM european conference on Computer Systems, pages 29-42. ACM, 2012.

[15] M. Pielot, K. Church, and R. de Oliveira. An in-situ study of mobile phone notifications. In Proceedings of the 16th international conference on Human-computer interaction with mobile devices \& services, pages 233-242. ACM, 2014.

[16] F. Qian, Z. Wang, A. Gerber, Z. Mao, S. Sen, and O. Spatscheck. Profiling resource usage for mobile applications: A cross-layer approach. In Proceedings of the 9th International Conference on Mobile Systems, Applications, and Services, MobiSys '11, pages 321-334, 2011.

[17] F. Qian, Z. Wang, A. Gerber, Z. M. Mao, S. Sen, and O. Spatscheck. Characterizing radio resource allocation for $3 \mathrm{~g}$ networks. In Proceedings of the 10th ACM SIGCOMM conference on Internet measurement, pages 137-150. ACM, 2010.

[18] F. Qian, Z. Wang, A. Gerber, Z. M. Mao, S. Sen, and O. Spatscheck. Top: Tail optimization protocol for cellular radio resource allocation. In Network Protocols (ICNP), 2010 18th IEEE International Conference on, pages 285-294. IEEE, 2010.

[19] R. R. Sokal and C. D. Michener. A Statistical Method for Evaluating Systematic Relationships. University of Kansas Scientific Bulletin, 38:1409-1438, 1958.

[20] L. Zhang, B. Tiwana, Z. Qian, Z. Wang, R. P. Dick, Z. M. Mao, and L. Yang. Accurate online power estimation and automatic battery behavior based power model generation for smartphones. In Proceedings of the eighth IEEE/ACM/IFIP international conference on Hardware/software codesign and system synthesis, pages 105-114. ACM, 2010. 